\title{
Analysis of Synchronization Impairments for Cooperative Base Stations Using OFDM
}

\author{
Konstantinos Manolakis, ${ }^{1}$ Christian Oberli, ${ }^{2}$ Volker Jungnickel, ${ }^{3}$ and Fernando Rosas ${ }^{4}$ \\ ${ }^{1}$ Huawei Technologies, European Research Center, 80892 Munich, Germany \\ ${ }^{2}$ Pontificia Universidad Católica de Chile, 7820436 Santiago, Chile \\ ${ }^{3}$ Technische Universität Berlin, 10623 Berlin, Germany \\ ${ }^{4}$ KU Leuven, 3001 Leuven, Belgium
}

Correspondence should be addressed to Konstantinos Manolakis; konstantinos.manolakis@huawei.com

Received 21 August 2014; Revised 10 February 2015; Accepted 12 February 2015

Academic Editor: Feifei Gao

Copyright (C) 2015 Konstantinos Manolakis et al. This is an open access article distributed under the Creative Commons Attribution License, which permits unrestricted use, distribution, and reproduction in any medium, provided the original work is properly cited.

\begin{abstract}
Base station cooperation is envisioned as a key technology for future cellular networks, as it has the potential to eliminate intercell interference and to enhance spectral efficiency. To date, there is still lack of understanding of how imperfect carrier and sampling frequency synchronization between transmitters and receivers limit the potential gains and what the actual system requirements are. In this paper, OFDM signal model is established for multiuser multicellular networks, describing the joint effect of multiple carrier and sampling frequency offsets. It is shown that the impact of sampling offsets is much smaller than the impact of carrier frequency offsets. The model is extended to the downlink of base-coordinated networks and closed-form expressions are derived for the mean power of users' self-signal, interuser, and intercarrier interference, whereas it is shown that interuser interference is the main source of degradation. The SIR is inverse to the base stations' carrier frequency variance and to the square of time since the last precoder update, whereas it grows with the number of base stations and drops with the number of users. Through user selection, the derived SIR upper bound can be approached. Finally, system design recommendations for meeting synchronization requirements are provided.
\end{abstract}

\section{Introduction}

Base station cooperation, also known as coordinated multipoint (CoMP), is an ambitious multiple-antenna technique, where antennas of multiple distributed base stations and those of multiple terminals served within those cells are considered as a distributed multiple-input multiple-output (MIMO) system [1-3]. In the downlink, also known as joint transmission (JT) CoMP, signal preprocessing at the base stations is applied to eliminate the intercell interference and to enhance the spectral efficiency. In the simplest case, data symbols are precoded with the pseudoinverse of the MIMO channel matrix; this method is known as zero-forcing (ZF) precoding [4]. Using ZF precoding, system performance becomes close to optimal in the high signal-to-noise ratio (SNR) regime, as shown in [5]. Deployment concepts for JT CoMP and field trial results have been reported in [6], whereas recent progress can be found in $[7,8]$. The role of
CoMP and integration aspects into next generation cellular systems are highlighted in [9]. Finally, an overview on cooperative communications can be found in [10].

The combination of MIMO techniques with orthogonal frequency division multiplexing (OFDM) has been a successful concept for broadband cellular networks and has enabled a significant increase of the spectral efficiency during the last years $[11,12]$. However, it quickly became clear that precise synchronization is vital for realizing the potential of MIMO-OFDM systems. It is known from [13] that the carrier frequency offset (CFO) causes intercarrier interference (ICI) as well as a phase drift on all OFDM subcarriers, known as common phase error (CPE). The sampling frequency offset (SFO) is also a source of ICI and implies a phase drift that grows linearly with frequency, thus affecting each subcarrier differently. Accurate maximum likelihood (ML) tracking algorithms have been developed and optimized for single-user point-to-point MIMO-OFDM in [14], whereas 
synchronization for multiuser MIMO within one cell has been studied in [15]. For the OFDM-based multiuser uplink, a signal model and compensation techniques have been developed in [16].

Considering distributed JT CoMP, cooperative base stations are located at different sites, which implies that their frequency up- and downconverters are driven by their own local oscillators, while sampling frequencies also differ among them. Signal modeling of JT CoMP with individual offsets in carrier and sampling frequencies in [17] revealed that orthogonality between multiple users' data signals is misaligned and interuser interference (IUI) arises. First insights into the performance degradation were obtained by numerical evaluation. In chapter 8 of [18], the sensitivity of CoMP to the CFO was analyzed for a scenario with two cooperating base stations. Similar observations have been reported in $[19,20]$, whereas methods for estimating multiple CFOs based on training signals have been developed in [21]. The problem of nonsynchronized cooperating base stations has been also investigated in [22-24], where the focus has been on how to estimate and compensate the multiple CFOs. In [25], propagation delay differences were also included for transmissions from distributed base stations with multiple CFOs. In [26], a scheme for synchronizing base stations has been proposed, based on a time-slotted roundtrip carrier synchronization protocol. The implementation of Global Positioning System- (GPS-) based synchronization for distributed base stations in an outdoor testbed has been reported by the authors in [27]. More recently, an overthe-air synchronization protocol has been proposed in [28], which is also applicable for networks with a large number of access points. A survey on physical layer synchronization for distributed wireless networks can be found in [29].

The first objective of the present paper is to investigate the synchronization requirements for base-coordinated multicellular MIMO networks. A major contribution of this work is the derivation of an exact signal model capturing the joint effect of multiple CFOs and SFOs at transmitters and receivers in a MIMO-OFDM system and over the time. Based on this model, it is shown that the impact of the SFO is negligible compared to the one of the CFO. Application of the model to the distributed CoMP downlink with ZF precoding leads to analytical closed-form expressions for the mean power of the users' self-signal, interuser, and intercarrier interferences. It is found that the interuser interference is the dominant source of signal degradation and that synchronization requirements for cooperating base stations are very high, compared to the ones in single-cell transmission. The mean signal-to-interference ratio (SIR) is analyzed and is approximately found to degrade quadratically with time and to be inversely proportional to the variance of the base stations' CFO. The SIR further grows with the number of base stations and drops with the number of users. In addition to the SIR analysis for the Rayleigh fading channel, an SIR upper bound is derived, which can be approached by appropriate user selection. Finally, recommendations for practical synchronization of distributed wireless networks are given.
The paper is organized as follows. In Section 2, a general signal model for a MIMO-OFDM communication system in the presence of multiple CFOs and SFOs is derived. In Section 3, the model is applied to the CoMP downlink and expressions are derived for mobile users' self-signal, interuser, and intercarrier interferences. Analysis in Section 4 leads to closed-form expressions for the mean power of the above signals and the resulting SIR. The system performance is evaluated analytically and verified by means of simulations in Section 5. Synchronization requirements are established and practical methods to fulfill them are discussed in Section 6. Finally, conclusions are summarized in Section 7.

\section{General Signal Model for Distributed MIMO-OFDM Systems}

In the following, a distributed MIMO network is considered with an arbitrary number of antenna branches at every base station and at every user. The cellular network uses OFDM for the air interface, with $N_{s}$ subcarriers, which are indexed with $k$ in the range $\left\{-N_{s} / 2, \ldots, N_{s} / 2-1\right\}$. An entire OFDM symbol is $N_{g}$ samples long, equal to $N_{s}$ samples plus the number of samples of the cyclic prefix. Integer $n$ indexes successive OFDM symbols and is hence a measure of time.

Each base station and each mobile are assumed to have their own carrier and sampling frequency, within typical ranges. The total number of transmit branches is $N_{t}$. Each transmit branch (can be a base station in the downlink or a user in the uplink), denoted by subindex $i$, has its individual sampling period $T_{i}$, carrier frequency $f_{i}$ and respective initial phase parameters $\tau_{i}$ and $\varphi_{i}$. In Figure 1, it is shown for a point-to-point transmission how sampling and carrier offsets misalign analog-to-digital and digital-to-analog conversion, as well as frequency conversion, respectively. The corresponding receiver parameters are denoted as $T_{j}, f_{j}, \tau_{j}$, and $\varphi_{j}$, while symbol $j$ is used for $\sqrt{-1}$. The digital modulation of subcarrier $k$ on transmit branch $i$ is represented by the complex-valued symbol $X_{i}(k)$. Due to different sampling timings between transmit branches of different base stations (downlink) or among mobile users (uplink), the intercarrier spacing is transmit-branch-specific and measures $\delta_{i}=$ $\left(N_{s} T_{i}\right)^{-1}$ Hertz. The ideal carrier frequency is denoted by $f_{c}$ and the ideal sampling period with $T$. For any transmitter or receiver, its $\mathrm{CFO}$ and $\mathrm{SFO}$ are defined as the the deviation from the ideal carrier frequency and sampling period, respectively. The complex baseband-equivalent frequency response of the passband channel between transmitter $i$ and receiver $j$ at frequency $f$ is denoted by $H_{j i}(f)$. It includes frequencyflat path loss and shadow fading as well as frequency-selective small-scale fading.

By following similar arguments as the ones leading to equation (8) in [14], and by considering the clarification in [30], that is, corrections in magnitude in order to keep signal energies consistent, it is found that the spectrum of the OFDM signal observed at any given receive branch $j$ has the form

$$
Y_{j}(f)=U_{j}(f, k)+\bar{U}_{j}(f, k)+N_{j}(f),
$$



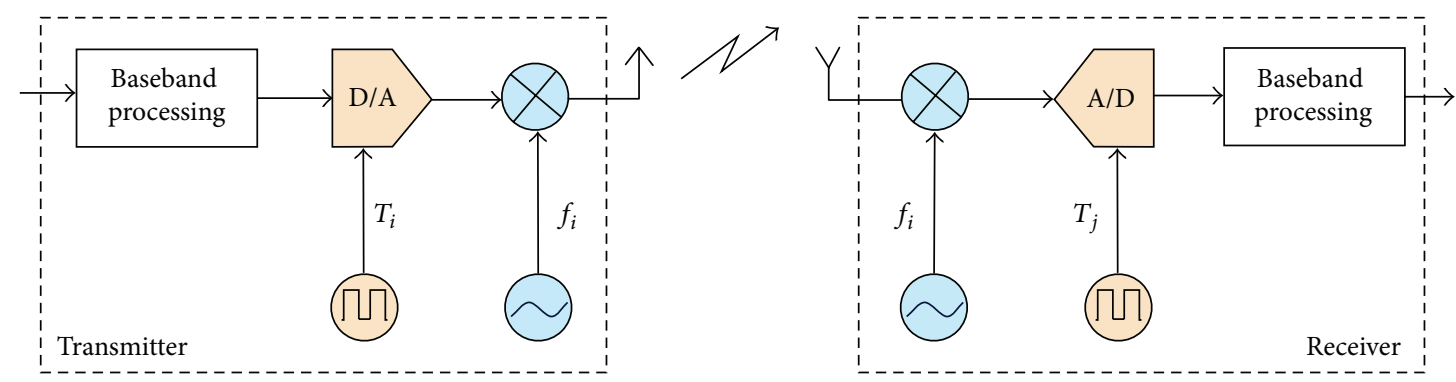

Figure 1: Transmitter and receiver have individual sampling periods $T_{i}$ and $T_{j}$ for digital-to-analog and analog-to-digital conversion and individual carrier frequencies $f_{i}$ and $f_{j}$ for upconversion and downconversion.

where $U_{j}(f, k)$ represents the continuous-frequency spectrum of the received multiuser signal at receive antenna $j$ for transmitted subcarrier $k$ (on-carrier signal). It is noted that $\bar{U}_{j}(f, k)$ is the received spectrum of the multiuser signal of all other subcarriers $v \neq k$, that is, the multiuser ICI (it is arbitrary which subcarrier is designated as $k$, but our analysis requires to single out one of them). The additive white Gaussian noise (AWGN) contributed by the front-end of receive antenna $j$ is denoted by $N_{j}(f)$. The above terms are given by

$$
\begin{array}{r}
U_{j}(f, k)=T_{j} \sum_{i=1}^{N_{t}} e^{\jmath\left(\varphi_{j}-\varphi_{i}\right)} \beta_{j i}(f, k) \\
\cdot X_{i}(k) H_{j i}\left(k \delta_{i}+f_{i}-f_{c}\right), \\
\bar{U}_{j}(f, k)=T_{j} \sum_{\nu=-N_{s} / 2}^{N_{s} / 2-1} \sum_{\substack{\nu \neq k \\
N_{t}}}^{\jmath\left(\varphi_{j}-\varphi_{i}\right)} \beta_{j i}(f, \nu) \\
\cdot X_{i}(\nu) H_{j i}\left(\nu \delta_{i}+f_{i}-f_{c}\right),
\end{array}
$$

with

$$
\begin{aligned}
\beta_{j i}(f, k)= & e^{-\jmath 2 \pi\left(f_{j}-f_{i}\right)\left(n N_{g} T_{j}+\tau_{j}\right)} \\
& \cdot e^{-j 2 \pi k \delta_{i}\left(n N_{g} T_{i}+\tau_{i}-n N_{g} T_{j}-\tau_{j}\right)} \\
& \cdot e^{-\jmath \pi\left(f+f_{j}-f_{i}-k \delta_{i}\right) T_{j}\left(N_{s}-1\right)} \\
& \cdot \frac{\sin \left[\pi\left(f+f_{j}-f_{i}-k \delta_{i}\right) T_{j} N_{s}\right]}{\sin \left[\pi\left(f+f_{j}-f_{i}-k \delta_{i}\right) T_{j}\right]} .
\end{aligned}
$$

The exponential terms in (4) are phase shifts due to carrier and sampling frequency misalignment, while the fractional term describes the loss of orthogonality among OFDM subcarriers, causing a leakage of the signal transmitted on subcarrier $k$. Note that $H_{j i}\left(k \delta_{i}+f_{i}-f_{c}\right)$ expresses the channel at the frequency of subcarrier $k$ plus a shift due to the transmitter's SFO and CFO.

The model given by (1) through (4) makes no assumption about the synchronization among branches and it is general for any MIMO-OFDM communication, also including cellular networks with base station cooperation. It describes how successive OFDM symbols are degraded under constant and uncompensated CFOs and SFOs as time goes by; that is, OFDM symbol index $n$ grows. The CFO must be smaller than half of a subcarrier spacing. For typical mobile receivers this implies that an earlier coarse frequency synchronization stage has succeeded, which we assume henceforth. Regarding SFO, the expressions are valid well beyond the typical range specified for SFO in commercial OFDM systems. It is to be noted, however, that, for a given SFO, the FFT window of each receiver branch does eventually drift away to a point at which intersymbol interference (ISI) arises between OFDM symbols. From that point on, severe degradation ensues and the model stops being valid. It is also implicit in our model that OFDM symbol timing has been acquired in a prior stage. The result assumes that the time dispersion of all the MIMO channel impulse responses, also from distributed base stations and mobile users, is shorter than the OFDM cyclic prefix in use.

Returning to the model, it can be observed in (2) and (3) that the phase offsets due to $\tau_{i}, \tau_{j}, \varphi_{i}$, and $\varphi_{j}$ may be considered, without loss of generality, as part of the channel. It follows that we may choose $\tau_{i}=0, \tau_{j}=0, \varphi_{i}=0$, and $\varphi_{j}=0$. We also point out that, in a practical implementation, for the $j$ th OFDM receiver branch, the output of its FFT corresponds to a sequence of samples of $Y_{j}(f)$ taken at frequencies $f=l / N_{s} T_{j}=l \delta_{j}$, where $l$ is the subcarrier index $\left(-N_{s} / 2 \leq l \leq N_{s} / 2-1\right)$ and $\delta_{j}$ is the receiver-side intercarrier spacing. Note that $l$ points to a slightly different frequency at each receive branch due to the different SFO and generally also to a different frequency than that pointed at by index $k$ at the various transmit branches $i$. Imposing the above conditions on (2) and (3), and focusing on an arbitrary received subcarrier $l=k$, we obtain the following discretefrequency signal model:

$$
Y_{j}(k)=U_{j}(k)+\bar{U}_{j}(k)+N_{j}(k),
$$

with

$$
\begin{gathered}
U_{j}(k)=\sum_{i=1}^{N_{t}} \beta_{j i}(k, k) X_{i}(k) H_{j i}(k), \\
\bar{U}_{j}(k)=\sum_{\substack{\nu=-N_{s} / 2 \\
\nu \neq k}}^{N_{s} / 2-1} \sum_{i=1}^{N_{t}} \beta_{j i}(k, v) X_{i}(\nu) H_{j i}(\nu),
\end{gathered}
$$




$$
\begin{aligned}
\beta_{j i}(k, v)= & e^{-\jmath 2 \pi n N_{g} T_{j}\left(f_{j}-f_{i}\right)} \\
& \cdot e^{-\jmath 2 \pi n N_{g} \nu \delta_{i}\left(T_{i}-T_{j}\right)} \\
& \cdot e^{-\jmath \pi\left(\left(N_{s}-1\right) / N_{s}\right)\left[\left(k-\nu\left(T_{j} / T_{i}\right)\right)+\left(f_{j}-f_{i}\right) T_{j} N_{s}\right]} \cdot \frac{1}{N_{s}} \\
& \cdot \frac{\sin \left[\pi\left(k-\nu\left(T_{j} / T_{i}\right)\right)+\pi\left(f_{j}-f_{i}\right) T_{j} N_{s}\right]}{\sin \left[\left(\pi / N_{s}\right)\left(k-v\left(T_{j} / T_{i}\right)\right)+\pi\left(f_{j}-f_{i}\right) T_{j}\right]},
\end{aligned}
$$

and with $N_{j}(k)$ being the receiver-side AWGN, of power $N_{0} \delta_{j}$. Note that in (6) and (7) we have approximated and defined $H_{j i}\left(k \delta_{i}+f_{i}-f_{c}\right) \approx H_{j i}\left(k \delta_{i}\right) \triangleq H_{j i}(k)$, because $\left|f_{i}-f_{c}\right|$ is assumed to be much smaller than the coherence bandwidth of the channel.

In practical system implementation, the carrier and sampling frequency clocks of a transmitter or receiver are derived from the same reference, that is, from the same local oscillator. Thus, for the product of an arbitrary $i$ th carrier frequency and sampling period $f_{i} \cdot T_{i} \triangleq \kappa$, it holds that $\kappa \gg 1$, as the (ideal) carrier frequency $f_{c}$ is two to three orders of magnitude larger than the (ideal) sampling frequency $1 / T$. The constant $\kappa$ depends only on the system and hardware design and is independent of $i$.

Considering this relationship in (8), it is straightforward to see that the exponent in the expression's second line, which captures the SFO effect on $\beta_{j i}(k, v)$ and which is maximized for $\nu=N_{s}$, still remains $\kappa$ times smaller than the exponent in the first line capturing the CFO effect. Similar findings can be observed comparing the influence of SFO with the one of the CFO onto the terms in the third and the fourth line of (8). Thus, it can be safely said that the impact of the SFO on $\beta_{j i}(k, v)$ is significantly weaker than the impact of the CFO, when using the same oscillator reference for both, at each individual transmitter and receiver.

The derivations up to here have been presented including both CFO and SFO for the sake of completeness, as well as for further reference. Expressions (5) through (8) provide the exact signal model, which characterizes the joint effect of multiple CFOs and SFOs over consecutive OFDM symbols in MIMO transmissions and is one important contribution of this work. However, beyond signal modeling, this work further aims to identify the major degradation sources, analyze the performance degradation, and determine from there the actual system requirements. To this end, in the following, we focus on the CFO and neglect the SFO, by assuming ideal sampling for all transmitters and receivers. With $T_{i}=T_{j}=T$, (8) becomes

$$
\begin{aligned}
\beta_{j i}(k, v)= & e^{-j 2 \pi\left[\left(f_{j}-f_{i}\right) t_{n}+\left(\left(N_{s}-1\right) / 2 N_{s}\right)(k-v)\right]} \\
& \cdot \frac{1}{N_{s}} \frac{\sin \left[\pi(k-v)+\pi\left(f_{j}-f_{i}\right) T N_{s}\right]}{\sin \left[\left(\pi / N_{s}\right)(k-v)+\pi\left(f_{j}-f_{i}\right) T\right]} .
\end{aligned}
$$

The discrete time variable $t_{n} \triangleq\left(n N_{g}+\left(N_{s}-1\right) / 2\right) T$ has been defined for convenience and is measured in seconds.

Expressions (5) to (9) provide the discrete-frequency signal model for a MIMO-OFDM communication system in the presence of multiple CFOs and SFOs as a function of time and will be used in the following section.

\section{Precoded Multicell Downlink Signal Model}

Now we specialize the model obtained in Section 2 to the CoMP downlink including joint precoding of the data signals. Here, the required channel state information (CSI) for the precoder calculation is estimated either at the base stations or at the terminals and fed back to the base stations, depending on whether time division duplex (TDD) or frequency division duplex (FDD) is used. For distributed base stations, coordination by means of a backhaul network is considered, which enables fast exchange of data and CSI.

It is to be noted that channel estimation errors and CSI delays due to the feedback and the backhaul network are not considered in this work. Their effect on JT CoMP is important and might even overwhelm the effects of imperfect synchronization, which we are analyzing here. A distinct analysis by the authors which includes the effect of imperfect channel knowledge and derives the resulting performance limitations can be found in [33]. We will therefore assume here that perfect knowledge of the downlink MIMO channel matrix is available at the base stations and is used for real-time precoding of the downlink signals. As already mentioned in Section 2, residual phase terms due to CFOs and SFOs are also considered as part of the (perfectly estimated) channel. We consider $N_{u}$ users, equipped with single-antenna terminals, which are jointly served by $N_{b}$ coordinated antenna branches among all base stations forming the cooperation cluster. There is no exchange of information between mobile users and out-of-cluster transmission is not considered in our model.

Transmissions are precoded on each OFDM subcarrier $k$ with the right-hand pseudoinverse of the $N_{u} \times N_{b}$ downlink MIMO channel matrix $\mathbf{H}(k)$ for that subcarrier, given by

$$
\mathbf{W}(k)=\mathbf{H}^{H}(k)\left[\mathbf{H}(k) \mathbf{H}^{H}(k)\right]^{-1} .
$$

The inverse is of size $N_{b} \times N_{u}$ and we assume it exists for $N_{b}>N_{u}$. Note that this condition can be met in practice by appropriate user selection and clustering of base stations, which is thereby assumed.

Next, consider $\mathbf{S}(k)$ to be an $N_{u} \times 1$ vector that contains the complex-valued data symbols $s_{u}(k)$ to be transmitted to the $N_{u}$ users on subcarrier $k$. Then, the $N_{b} \times 1$ vector $\mathbf{X}(k)$ of precoded symbols $X_{b}(k)$ to be transmitted on subcarrier $k$ by the $N_{b}$ base stations is $\mathbf{X}(k)=\mathbf{W}(k) \mathbf{S}(k)$, where elements are given by

$$
X_{b}(k)=\sum_{u=1}^{N_{u}} w_{b u}(k) s_{u}(k),
$$

and $w_{b u}(k)$ are the elements of $\mathbf{W}(k)$. Transmitter index $i$ has been replaced with $b$ to stress that, from here on, transmitters are base stations. Imposing the expression of the precoded symbol (11) into $U_{j}(k)$ given by (6), we obtain the on-carrier signal on subcarrier $k$ of user $j$, given by

$$
U_{j}(k)=\check{s}_{j}(k)+\bar{s}_{j}(k),
$$


with

$$
\begin{gathered}
\check{s}_{j}(k)=s_{j}(k) \sum_{b=1}^{N_{b}} \beta_{j b}(k, k) H_{j b}(k) w_{b j}(k), \\
\bar{s}_{j}(k)=\sum_{\substack{u=1 \\
u \neq j}}^{N_{u}} s_{u}(k) \sum_{b=1}^{N_{b}} \beta_{j b}(k, k) H_{j b}(k) w_{b u}(k) .
\end{gathered}
$$

An arbitrary user $j$ has been singled out in (13) from the remaining users. Thus, $\check{s}_{j}(k)$ represents the self-signal, while $\bar{s}_{j}(k)$ represents the IUI observed by user $j$, given by (14). This interference is due to the loss of orthogonality of the precoded transmission to multiple users, caused by synchronization impairments. Imposing (11) into $\bar{U}_{j}(k)$ in (7), we obtain the ICI on subcarrier $k$ of user $j$, given by

$$
\bar{U}_{j}(k)=\sum_{\substack{v=-N_{s} / 2 \\ \nu \neq k}}^{N_{s} / 2-1} \sum_{u=1}^{N_{u}} s_{u}(\nu) \sum_{b=1}^{N_{b}} \beta_{j b}(k, v) H_{j b}(v) w_{b u}(v) .
$$

Our complete discrete-frequency CoMP downlink signal model is then

$$
Y_{j}(k)=\check{s}_{j}(k)+\bar{s}_{j}(k)+\bar{U}_{j}(k)+N_{j}(k),
$$

with $\check{s}_{j}(k), \bar{s}_{j}(k)$, and $\bar{U}_{j}(k)$ given by (13), (14), and (15), respectively, and $N_{j}(k)$ being the AWGN of power $N_{0} \delta_{j}$. It is noted that the model of Section 3 is valid independently if for $\beta_{j i}(k, v)$ the exact expression (8) or its simplification (9) is used, where the SFO is neglected.

\section{Analysis of Signal and Interference Powers}

The following section contains an in-depth analysis of the impact of synchronization impairments onto the performance. It is organized as follows: first, we study the power of a user's self-signal (useful signal) and then the IUI and ICI. Next, we show that ICI is small compared to IUI and provide analytical expressions for the mean SIR. Finally, we highlight the value of user selection and show its impact onto the performance.

Conceptually, the rise of IUI and ICI due to imperfect synchronization can be understood as a dispersion of the energy allocated on a specific subcarrier of a specific user to other users (IUI) and to other subcarriers (ICI). This implies a drop of the self-signal power and a rise, on that user and that subcarrier, of the power of IUI and ICI from other users' and other subcarriers' losses. In what follows, we proceed under the assumption that data symbols are statistically independent between users and across subcarriers; that is, $\mathbb{E}\left\{s_{j}(k) s_{u}^{*}(v)\right\}=E_{s} \delta_{j u} \delta_{k v}$, where $\mathbb{E}\{\cdot\}$ denotes the expectation operator, $E_{s}$ the mean energy per data symbol, and $\delta_{x y}$ the Kronecker delta between $x$ and $y$.

4.1. Power of the User's Self-Signal. Since there is statistical independence between data symbols, channel coefficients, and synchronization parameters, the mean power of the user's self-signal (13) is

$$
\mathbb{E}\left\{\left|\check{s}_{j}\right|^{2}\right\}=E_{s} \sum_{b_{1}=1}^{N_{b}} \sum_{b_{2}=1}^{N_{b}} \mathbb{E}\left\{\beta_{j b_{1}} \beta_{j b_{2}}^{*}\right\} \mathbb{E}\left\{H_{j b_{1}} w_{b_{1} j} H_{j b_{2}}^{*} w_{b_{2} j}^{*}\right\},
$$

where subcarrier index $k$ has been omitted for simplicity of notation and cross-terms equal to zero have been already disregarded.

In a first step, we analyze $\mathbb{E}\left\{\beta_{j b_{1}} \beta_{j b_{2}}^{*}\right\}$, which appears in (17). Using therefore $v=k$ in (9) and replacing transmitter index $i$ with base station index $b$, we reach

$$
\beta_{j b}=\frac{1}{N_{s}} \cdot e^{-j 2 \pi\left(f_{j}-f_{b}\right) t_{n}} \cdot \frac{\sin \left[\pi\left(f_{j}-f_{b}\right) T N_{s}\right]}{\sin \left[\pi\left(f_{j}-f_{b}\right) T\right]},
$$

which does not depend on the subcarrier index $k$. By noting that $\left|f_{j}-f_{b}\right| \ll 1 / T$, we can safely say that the argument $x=$ $\pi\left(f_{j}-f_{b}\right) T$ of the $\sin (x)$ in the denominator of the fraction on the right-hand side of (18) is very small. The same term also appears in the exponential term, and both multiplicative terms in (18) are close to one. But, comparing the magnitude deviations from unity, we see that for typical synchronization parameters $\left|1-\left(1 / N_{s}\right)\left(\sin \left(N_{s} x\right) / \sin (x)\right)\right| \ll \mid 1-$ $e^{-\jmath(2.14 n+1) N_{s} x} \mid$ (this results in $N_{s} \gg 1$ and a cyclic prefix equal to $0.07 N_{s}$ [31]). In absolute terms, we can further use the first order Taylor series expansion $\left(1 / N_{s}\right)\left(\sin \left(N_{s} x\right) / \sin (x)\right) \approx 1$ and reach

$$
\beta_{j b_{1}} \beta_{j b_{2}}^{*} \approx e^{-j 2 \pi\left(f_{j}-f_{b_{1}}\right) t_{n}} \cdot e^{j 2 \pi\left(f_{j}-f_{b_{2}}\right) t_{n}}=e^{j 2 \pi\left(f_{b_{1}}-f_{b_{2}}\right) t_{n}} .
$$

It is interesting to observe that the power of the exponential term, as approximated in (19), depends on the difference between the base stations' carrier frequencies. The power loss effect of the symbol transmitted on subcarrier $k$, which is the generating factor of ICI, depends on both the CFOs of the base stations and of the mobile users. The effect that has been neglected here due to its relatively small role compared to the one of the exponential term will be however thoroughly analyzed in Section 4.2.

For transmission from one base station, that is, for case $b_{1}=b_{2}$, it is immediate that (19) equals one. For $b_{1} \neq b_{2}$, it is reasonable to assume that different base stations' carrier frequencies $f_{b_{1}}$ and $f_{b_{c}}$ are statistically independent, which allows for expressing the expectation of (19) as a product of two terms, each depending on one base station's CFO:

$$
\mathbb{E}\left\{\beta_{j b_{1}} \beta_{j b_{2}}^{*}\right\}=\mathbb{E}\left\{e^{j 2 \pi\left(f_{b_{1}}-f_{c}\right) t_{n}}\right\} \cdot \mathbb{E}\left\{e^{-j 2 \pi\left(f_{b_{2}}-f_{c}\right) t_{n}}\right\} .
$$

By assuming further that the CFOs are identically distributed, the second term of the right-hand side in (20) is the complexconjugate of the first one; hence (20) can be developed as

$$
\begin{aligned}
\mathbb{E}\left\{\beta_{j b_{1}} \beta_{j b_{2}}^{*}\right\} & =\left|\mathbb{E}\left\{e^{-j 2 \pi\left(f_{b}-f_{c}\right) t_{n}}\right\}\right|^{2} \\
& =\left|\int p_{\left(f_{b}-f_{c}\right)} e^{-j 2 \pi\left(f_{b}-f_{c}\right) t_{n}} d\left(f_{b}-f_{c}\right)\right|^{2},
\end{aligned}
$$


where $p_{\left(f_{b}-f_{c}\right)}$ denotes the probability distribution function (pdf) of the CFO $\left(f_{b}-f_{c}\right)$ and is independent on index $b$. It is to be noted that the integral in (21) is a Fourier transform of the CFO's pdf. Hence, we can write

$$
\mathbb{E}\left\{\beta_{j b_{1}} \beta_{j b_{2}}^{*}\right\}= \begin{cases}1, & b_{1}=b_{2} \\ \left|\mathscr{F}_{p}\left(t_{n}\right)\right|^{2}, & b_{1} \neq b_{2},\end{cases}
$$

where $\mathscr{F}_{p}(\cdot)$ denotes the Fourier transform of $p_{\left(f_{b}-f_{c}\right)}$ and is here a function of time. For being a characteristic function, that is, the Fourier transform of a pdf, it is guaranteed that $\left|\mathscr{F}_{p}\left(t_{n}\right)\right| \leq 1$ (see [32]), which means that the power of the user's self-signal (17) drops under imperfect carrier synchronization conditions.

Result (22) can be used for developing (17) further by separating the sum over $b_{2}$ into the cases $b_{2}=b_{1}$ and $b_{2} \neq b_{1}$ :

$$
\begin{aligned}
\mathbb{E}\left\{\left|\check{s}_{j}\right|^{2}\right\}= & E_{s} \cdot \sum_{b=1}^{N_{b}} \mathbb{E}\left\{\left|H_{j b} w_{b j}\right|^{2}\right\}+E_{s} \cdot\left|\mathscr{F}_{p}\left(t_{n}\right)\right|^{2} \\
& \cdot \sum_{b_{1}=1}^{N_{b}} \sum_{\substack{b_{2}=1 \\
b_{2} \neq b_{1}}}^{N_{b}} \mathbb{E}\left\{H_{j b_{1}} H_{j b_{2}}^{*} w_{b_{1} j} w_{b_{2} j}^{*}\right\} \\
= & E_{s} \cdot\left(1-\left|\mathscr{F}_{p}\left(t_{n}\right)\right|^{2}\right) \cdot \sum_{b=1}^{N_{b}} \mathbb{E}\left\{\left|H_{j b} w_{b j}\right|^{2}\right\} \\
& +E_{s} \cdot\left|\mathscr{F}_{p}\left(t_{n}\right)\right|^{2} \cdot \sum_{b_{1}=1}^{N_{b}} \sum_{b_{2}=1}^{N_{b}} \mathbb{E}\left\{H_{j b_{1}} H_{j b_{2}}^{*} w_{b_{1} j} w_{b_{2} j}^{*}\right\} .
\end{aligned}
$$

The double sum in the last line in (23) can be formulated as

$$
\begin{aligned}
\sum_{b_{1}=1}^{N_{b}} \sum_{b_{2}=1}^{N_{b}} \mathbb{E}\left\{H_{j b_{1}} H_{j b_{2}}^{*} w_{b_{1} j} w_{b_{2} j}^{*}\right\} & \\
= & \mathbb{E}\left\{\sum_{b_{1}=1}^{N_{b}} H_{j b_{1}} w_{b_{1} j} \cdot \sum_{b_{2}=1}^{N_{b}} H_{j b_{2}}^{*} w_{b_{2} j}^{*}\right\},
\end{aligned}
$$

which, by considering the ZF condition

$$
\sum_{b=1}^{N_{b}} H_{j b} w_{b u}=\delta_{j u}
$$

is found to be equal to 1 . Thus, (23) can be written as

$$
\mathbb{E}\left\{\left|\check{s}_{j}\right|^{2}\right\}=E_{s}\left[1-\left(1-\left|\mathscr{F}_{p}\left(t_{n}\right)\right|^{2}\right) K_{\mathrm{U}}\right]
$$

where we defined $K_{\mathrm{U}}=1-\sum_{b=1}^{N_{b}} \mathbb{E}\left\{\left|H_{j b} w_{b j}\right|^{2}\right\}$. Note that (25) cannot be applied to the sum terms in the first and third line of (23) because of the power index. The constant $K_{U}$ depends on the precoder and the channel statistical properties. As shown in Appendix A for ZF precoding, given a channel matrix with $N_{b}>N_{u}$ and complex Gaussian independent and identically distributed (i.i.d.) entries (Rayleigh fading channel), with zero mean and variance $\sigma_{h}^{2}, K_{\mathrm{U}}$ can be approximated as

$$
K_{\mathrm{U}} \approx 1-\frac{1}{N_{b}-N_{u}} .
$$

If we further consider the case in which the CFOs of the base stations are Gaussian i.i.d. with zero mean and variance $\sigma_{f}^{2}$, the Fourier transform in (22) becomes

$$
\left|\mathscr{F}_{p}\left(t_{n}\right)\right|^{2}=e^{-4 \pi^{2} \sigma_{f}^{2} t_{n}^{2}} \approx 1-4 \pi^{2} \sigma_{f}^{2} t_{n}^{2},
$$

where the first order Taylor series expansion $e^{-x} \approx 1-x$ has been used. It is to be noted that this approximation is accurate for typical values of $\sigma_{f}$ and $t_{n}$. Using approximations (27) and (28), we can formulate the mean power of the user's self-signal (26) as

$$
\mathbb{E}\left\{\left|\check{s}_{j}\right|^{2}\right\} \approx E_{s}\left[1-4 \pi^{2} \sigma_{f}^{2} t_{n}^{2} \cdot\left(1-\frac{1}{N_{b}-N_{u}}\right)\right],
$$

from where we see that it decreases linearly with the base stations' CFO variance and quadratically with time. Result (29) also shows that the mean power of the self-signal grows with the number of base stations and drops with the number of users. It is noteworthy that, for $N_{u}=N_{b}-1$, the mean power of the self-signal remains constant. However, this should not be used as a system design rule; as for determining the system performance, the degradation due to IUI and ICI must be considered as well.

4.2. Power of the Interuser Interference. The derivation of the mean power of the IUI (14) follows similar steps as the ones in Section 4.1. Concretely,

$$
\begin{aligned}
& \mathbb{E}\left\{\left|\bar{s}_{j}\right|^{2}\right\}= E_{s} \cdot \sum_{\substack{u=1 \\
u \neq j}}^{N_{u}} \sum_{b_{1}=1}^{N_{b}} \sum_{b_{2}=1}^{N_{b}} \mathbb{E}\left\{\beta_{j b_{1}} \beta_{j b_{2}}^{*}\right\} \\
& \cdot \mathbb{E}\left\{H_{j b_{1}} w_{b_{1} u} H_{j b_{2}}^{*} w_{b_{2} u}^{*}\right\},
\end{aligned}
$$

and noting that in this case always $j \neq u$ in (25), we find that

$$
\mathbb{E}\left\{\left|\bar{s}_{j}\right|^{2}\right\}=E_{s}\left(1-\left|\mathscr{F}_{p}\left(t_{n}\right)\right|^{2}\right) K_{\mathrm{IUI}},
$$

where $K_{\text {IUI }}=\sum_{u=1, u \neq j}^{N_{u}} \sum_{b=1}^{N_{b}} \mathbb{E}\left\{\left|H_{j b} w_{b u}\right|^{2}\right\}$. If all users undergo identical channel statistics, $K_{\mathrm{IUI}}$ simplifies to $K_{\mathrm{IUI}}=\left(N_{u}-1\right)$. $\sum_{b=1}^{N_{b}} \mathbb{E}\left\{\left|H_{j b} w_{b u}\right|^{2}\right\}$, whereas using the results of Appendix A for the Rayleigh fading channel and $N_{b}>N_{u}$, we find

$$
K_{\mathrm{IUI}} \approx \frac{N_{u}-1}{N_{b}-N_{u}} .
$$

Using (32) and (28) in (31), we obtain

$$
\mathbb{E}\left\{\left|\bar{s}_{j}\right|^{2}\right\} \approx E_{s} \cdot 4 \pi^{2} \sigma_{f}^{2} t_{n}^{2} \cdot \frac{N_{u}-1}{N_{b}-N_{u}} .
$$

Result (33) reveals that the IUI power grows linearly with the base stations' CFO variance and quadratically with time. 
Using more base stations decreases the IUI power, while adding more users results in increasing it. Due to the approximation used in (19), it can be said that the impact of the mobile users' CFO onto the IUI power level is less significant than the impact of the base stations' CFOs.

4.3. Power of the Intercarrier Interference. Following similar steps as before, now for (15), the mean power of the ICI is

$$
\begin{gathered}
\mathbb{E}\left\{\left|\bar{U}_{j}(k)\right|^{2}\right\} \\
=E_{s} \cdot \sum_{\substack{\nu=-N_{s} / 2 \\
\nu \neq k}}^{N_{s} / 2-1} \sum_{u=1}^{N_{u}} \sum_{b_{1}=1}^{N_{b}} \sum_{b_{2}=1}^{N_{b}} \mathbb{E}\left\{\beta_{j b_{1}}(k, \nu) \beta_{j b_{2}}^{*}(k, \nu)\right\} \\
\cdot \mathbb{E}\left\{H_{j b_{1}}(\nu) w_{b_{1} u}(\nu) H_{j b_{2}}^{*}(\nu) w_{b_{2} u}^{*}(\nu)\right\} .
\end{gathered}
$$

Assuming identical channel statistics for all subcarriers, the last term in (34) does not depend on subcarrier index $v$ and becomes $\mathbb{E}\left\{H_{j b_{1}} w_{b_{1} u} H_{j b_{2}}^{*} w_{b_{2} u}^{*}\right\}$, already seen in Sections 4.1 and 4.2. For convenience we define

$$
\sum_{\substack{\nu=-N_{s} / 2 \\ v \neq k}}^{N_{s} / 2-1} \mathbb{E}\left\{\beta_{j b_{1}}(k, v) \beta_{j b_{2}}^{*}(k, v)\right\} \triangleq \begin{cases}B_{1}, & b_{1}=b_{2} \\ B_{2}, & b_{1} \neq b_{2},\end{cases}
$$

which is analyzed in Appendix B. In (34), we separate the sum over $b_{2}$ into the cases $b_{2}=b_{1}$ and $b_{2} \neq b_{1}$ as in (23) and obtain

$$
\begin{aligned}
\mathbb{E}\left\{\left|\bar{U}_{j}(k)\right|^{2}\right\} & E_{s} \cdot B_{1} \cdot \sum_{u=1}^{N_{u}} \sum_{b=1}^{N_{b}} \mathbb{E}\left\{\left|H_{j b} w_{b u}\right|^{2}\right\} \\
& +E_{s} \cdot B_{2} \cdot \sum_{u=1}^{N_{u}} \sum_{b_{1}=1}^{N_{b}} \sum_{b_{2}=1}^{N_{b}} \mathbb{E}\left\{H_{j b_{1}} w_{b_{1} u} H_{j b_{2}}^{*} w_{b_{2} u}^{*}\right\} \\
= & E_{s} \cdot\left(B_{1}-B_{2}\right) \cdot \sum_{u=1}^{N_{u}} \sum_{b=1}^{N_{b}} \mathbb{E}\left\{\left|H_{j b} w_{b u}\right|^{2}\right\} \\
& +E_{s} \cdot B_{2} \cdot \sum_{u=1}^{N_{u}} \sum_{b_{1}=1}^{N_{b}} \sum_{b_{2}=1}^{N_{b}} \mathbb{E}\left\{H_{j b_{1}} w_{b_{1} u} H_{j b_{2}}^{*} w_{b_{2} u}^{*}\right\} .
\end{aligned}
$$

We use the expressions for $B_{1}$ and $B_{2}$ in Appendix B, which provide accurate approximations for Gaussian distributed CFOs for the base stations and the mobile user $j$ with zero mean and variances $\sigma_{f}^{2}$ and $\sigma_{f, j}^{2}$, respectively, and reach

$$
\mathbb{E}\left\{\left|\bar{U}_{j}(k)\right|^{2}\right\} \approx \frac{E_{s} \pi^{2}}{3 \delta^{2}} \cdot\left(\sigma_{f}^{2} \cdot K_{\mathrm{ICI}}+\sigma_{f, j}^{2}\right) .
$$

Here, $K_{\mathrm{ICI}}=\sum_{u=1}^{N_{u}} \sum_{b=1}^{N_{b}} \mathbb{E}\left\{\left|H_{j b} w_{b u}\right|^{2}\right\}=K_{\mathrm{IUI}}-K_{\mathrm{U}}+1$ is a constant. For a Rayleigh fading MIMO channel, we use in (37) the expression provided by Appendix A for $K_{\mathrm{ICI}}$ and reach

$$
\mathbb{E}\left\{\left|\bar{U}_{j}(k)\right|^{2}\right\} \approx \frac{E_{s} \pi^{2}}{3 \delta^{2}} \cdot\left(\sigma_{f}^{2} \cdot \frac{N_{u}}{N_{b}-N_{u}}+\sigma_{f, j}^{2}\right) .
$$

Expression (38) shows that the mean power of the user's ICI can be split into two additive parts: the first part depending on the base stations' CFO variance, which is multiplied with a weighting factor $K_{\text {ICI }}$ according to the cluster size, and the second part depending on the user's own CFO. Furthermore, (38) reveals that the power of the ICI does not vary with time and that it is inverse to the square of the OFDM subcarrier spacing.

4.4. ICI-to-IUI Ratio and SIR. The relative magnitudes of the power terms derived so far in this section are analyzed next. A simple expression for the mean ICI-to-IUI power ratio of a user $j$ (an interference-to-interference ratio, IIR) can be obtained from (33) and (38):

$$
\operatorname{IIR}=\frac{\mathbb{E}\left\{\left|\bar{U}_{j}\right|^{2}\right\}}{\mathbb{E}\left\{\left|\bar{s}_{j}\right|^{2}\right\}} \approx \frac{1}{12 \delta^{2} t_{n}^{2}} \cdot\left(\frac{N_{u}}{N_{u}-1}+\xi^{2} \frac{N_{b}-N_{u}}{N_{u}-1}\right),
$$

with $N_{b}>N_{u} \geq 2$ and $\xi=\sigma_{f, j} / \sigma_{f}$ defined as the ratio between the standard deviations of the user's and the base stations' CFOs. By replacing $\delta=\left(N_{s} T\right)^{-1}$ and discrete time $t_{n}=(1.07 n+0.5) N_{s} T$ (this results in $N_{s} \gg 1$ and a cyclic prefix equal to $0.07 N_{s}[33]$ ), relation (39) becomes

$$
\operatorname{IIR} \approx \frac{1}{12(1.07 n+0.5)^{2}} \cdot\left(\frac{N_{u}}{N_{u}-1}+\xi^{2} \frac{N_{b}-N_{u}}{N_{u}-1}\right) .
$$

It is interesting to observe that, in the approximation (40), the IIR does not depend on the OFDM system parameters but only on the OFDM symbols index $n$.

Regarding the ratio $\xi$, it has been shown in $[14,21]$ that mobile terminals attain a carrier frequency accuracy, which is at least one order of magnitude below the accuracy of typical base station oscillators used in Third Generation Partnership Project (3GPP) Long Term Evolution (LTE) [31]. Assuming, for instance, $\xi^{2} \gg 1$, the first additive term in (40) becomes much smaller than the second term, indicating that the terminals' CFO is the main source of ICI. Using $\xi=0$, one can evaluate the IIR including only the ICI part due to the base stations' CFOs; in this case, the ratio does not depend on the base stations' CFOs at all. Using a more practical value of, for example, $\xi=10$, the largest possible IIR after $n=14$ OFDM symbols $\left(t_{n} \approx 1 \mathrm{~ms}\right)$, which occurs with $N_{u}=2$ users given $N_{b}=7$, equals $-7.3 \mathrm{~dB}$. The IIR drops quickly, down already to $-27.3 \mathrm{~dB}$ for $n=140\left(t_{n} \approx 10 \mathrm{~ms}\right)$. These quantitive results show that for typical JT CoMP scenarios, the IUI dominates over the ICI.

We will now neglect the ICI and define the mean SIR (selfuser signal-to-IUI ratio) by the ratio between (26) and (31):

$$
\operatorname{SIR}=\frac{\left|\mathscr{F}_{p}\left(t_{n}\right)\right|^{2}}{\left(1-\left|\mathscr{F}_{p}\left(t_{n}\right)\right|^{2}\right) K_{\mathrm{IUI}}}-\frac{1}{N_{u}-1} .
$$

For a MIMO channel with i.i.d. Rayleigh fading entries $\left(K_{\text {IUI }}\right.$ given by (32)) and for i.i.d. Gaussian CFOs $\left(\left|\mathscr{F}_{p}\left(t_{n}\right)\right|^{2}\right.$ given by (28)), (41) can be simplified as

$$
\operatorname{SIR} \approx \frac{1}{4 \pi^{2} \sigma_{f}^{2} t_{n}^{2}} \cdot \frac{N_{b}-N_{u}}{N_{u}-1}-\frac{N_{b}-N_{u}+1}{N_{u}-1} .
$$


Expressions (41) and (42) are valid for $N_{b}>N_{u} \geq 2$. Expression (42) reveals that, for ZF precoding, the mean SIR is in an approximately inverse relation to the base stations' CFO variance and to the square of time. Furthermore, it is shown that the SIR grows with the number of base stations and drops with the number of jointly served users.

\subsection{The Value of User Selection in JT CoMP and an SIR Bound.} The SNR gains in JT CoMP are increased if a scheduler selects the appropriate users to be jointly served on the same time and frequency resources. As shown in [34], the selection criteria are based on the rule that all users gain from the cooperation in the cluster, compared to the case of noncoordinated transmission. Evaluated on a field scenario, the resulting singular value statistics after such user selection was found to be comparable to the one of a Rayleigh fading channel, a fact that also relates to scenarios where users are located close to the cell edge, that is, in which gains through coordination are particularly large.

Considering now an idealized scenario, from the precoding point of view, with orthogonal channel vectors of equal power among the users, scenario which has been analyzed in $[5,11]$, an upper bound can be derived for the mean SIR using ZF precoding in JT CoMP. Using the expression for $K_{\mathrm{IUI}}$ as provided in Appendix A for $N_{b}>N_{u} \geq 2$, we obtain the following expression:

$$
\operatorname{SIR}_{\max } \approx \frac{1}{4 \pi^{2} \sigma_{f}^{2} t_{n}^{2}} \cdot \frac{N_{b}}{N_{u}-1}-\frac{N_{b}+1}{N_{u}-1}
$$

The distance between (42) and (43) reveals the potential for SIR enhancement, if the users' selection process considers the orthogonality among their channel vectors.

It should be clarified at this point that (42) and (43) do not consider any gains from resource allocation, the evaluation of which not in the scope of this work. In an orthogonal frequency division multiple access (OFDMA) system, each user can be assigned a part of the spectrum in a way that his performance and the network performance can be optimized, as known from [35] and also shown in [36] for the multiuser downlink.

The signal model given in Section 3 for the impaired OFDM-based JT CoMP is valid for any OFDMA scheme. Using OFDMA in the downlink does not affect the signal structure of a user's received self-signal and the IUI. The ICI also has the same structure and statistical properties, as typically data are transmitted on all subcarriers, which is recommended for keeping frequency-flat distribution of the ICI power [13]. The degradation mechanisms due to multiple CFOs and SFOs, as described in our model, will be the same for any system using OFDM.

What indeed changes in OFDMA is the channel statistics for each user after resource allocation. Therefore, if intended to evaluate the overall performance of a coordinated multipoint system using OFDMA, the general mean power expressions (26), (31), and (37) would need to be evaluated for the actual users' channel statistics. This procedure practically implies a calculation of constants $K_{\mathrm{U}}, K_{\mathrm{IUI}}$, and $K_{\mathrm{ICI}}$.
In this section, the received power of a user was studied in relation to the interference from other users and other subcarriers, in a multicellular multiuser downlink with cooperative base stations. Closed-form expressions and accurate approximations for all relevant terms were derived. Moreover, it was demonstrated that interuser interference has the most relevant effect. Finally, the impact of the radio channel was investigated and analytical SIR expressions were derived for zero-forcing precoding.

\section{Evaluation and Numerical Validation}

In this section, analytical results of Section 4 are evaluated and verified by simulations. Based on those results, adequate requirements are determined for the base stations' oscillators in CoMP systems.

A JT CoMP scenario is considered, where a cooperation cluster of $N_{b}=7$ base stations transmits jointly by using zero-forcing precoding to $N_{u}$ terminals on the same time and frequency resource, with $N_{b}>N_{u} \geq 2$. Out-of-cluster interference is not considered.

At time instant $t=0$, the base stations receive an update of the downlink channel matrix and compute a precoding matrix. This will be used during the following $10 \mathrm{~ms}$, time at which a new channel update will be obtained and a new precoder will be calculated. This routine agrees with the current 3GPP LTE channel and precoder updating cycle [31]. As already mentioned in Section 3, it is assumed that the radio channel is perfectly estimated and available at the base stations at $t=0$, whereas channel aging effects are not considered either. Between updating instants, the phases of the local base stations' oscillators slowly drift away from each other because of the individual frequency offsets with respect to the ideal carrier frequency $f_{c}$. As a consequence, self-signal drops, IUI grows, and overall the SIR drops with time. In a practical system, each user can use downlink pilot symbols to estimate and equalize its own CFO and avoid ICI enhancement. However, mobiles cannot stop the IUI from rising, because the degradation is caused jointly by all the misaligned base station oscillators.

The oscillator accuracy Osc, typically specified in parts per million ( $\mathrm{ppm}$ ) or parts per billion ( $\mathrm{ppb})$, relates to the standard deviation of the resulting carrier frequency as $\sigma_{f}=$ Osc $\cdot f_{c}$. The CFO variation over time is assumed to be slow enough to be considered static with respect to the signaling and precoder updating timescale. Here, Gaussian i.i.d. CFOs are randomly assigned to base stations and mobile users, all with zero mean and standard deviations given by $\sigma_{f}$ and $\sigma_{f, j}$, respectively.

Typical 3GPP LTE parameters are used, specified in [31]. The broadband OFDM signal has $N_{s}=2048$ subcarriers, separated by $\delta=15 \mathrm{kHz}$. The length of the cyclic prefix is 144 samples, resulting in OFDM symbols with a length of $N_{g}=$ 2192 samples. The ideal carrier and sampling frequencies are $f_{c}=2.65 \mathrm{GHz}$ and $1 / T=30.72 \mathrm{MHz}$, respectively. The energy per data symbol is set to $E_{s}=1$.

Figure 2 depicts the mean power of the IUI and ICI over time passed from the last precoder update (in logarithmic scale), for 3 and 6 mobile users, a base station oscillator 


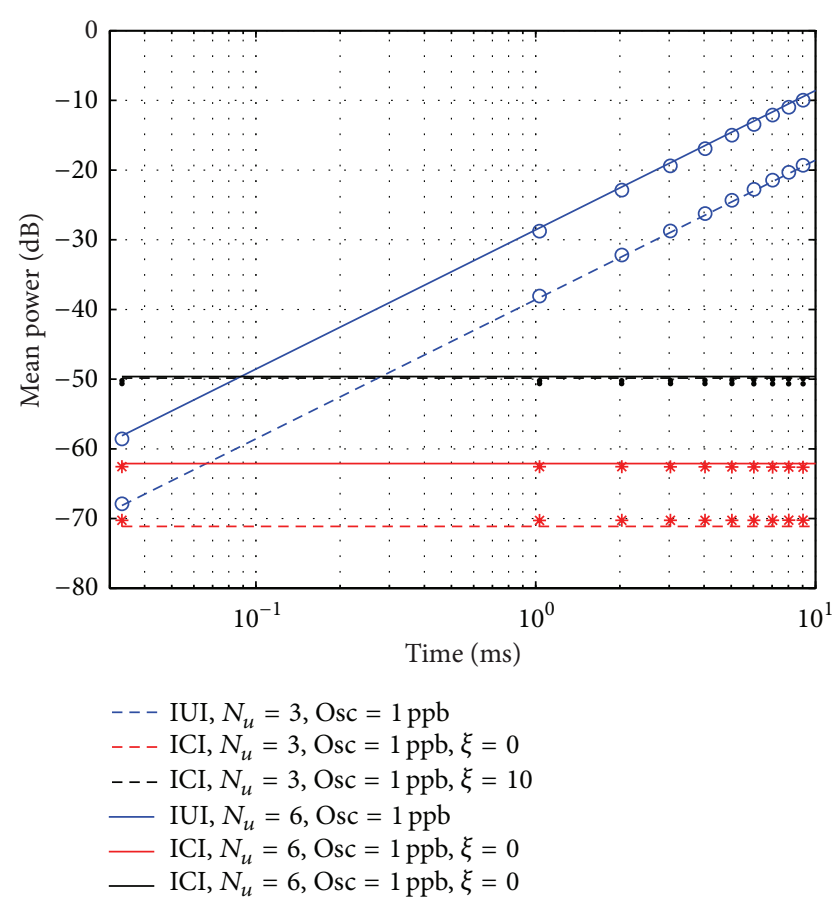

FIGURE 2: Mean power of interuser and intercarrier interferences in a Rayleigh fading channel. Here, 7 base stations serve 3 and 6 users, respectively. Analytical results are shown by lines, while markers show numerical evaluations.

accuracy of $1 \mathrm{ppb}$, and Rayleigh fading channel conditions. The curves illustrate analytical results given by (33) and (38), whereas solid lines depict results for 3 users and dashed lines for 6 users, respectively. Markers show respective numerical evaluations of the mean power of (14) and (15) over $10^{3}$ independent realizations of the MIMO channel matrix entries with $\sigma_{h}^{2}=1$ and of the bases' CFOs. Regarding the ICI, two scenarios are considered: a scenario with $\xi=$ 0 , that is, perfectly synchronized mobile users, in order to evaluate solely the ICI due to the base stations' CFOs (red) and a second scenario with nonsynchronized mobiles, with a CFO accuracy of an order of magnitude lower than the base stations' one, that is, $\xi=10$ (black), where the total ICI is evaluated.

First of all, Figure 2 verifies the analytical expressions (including mathematical approximations) by numerical means. It is further observed that the IUI grows approximately with the square of time and that it increases with the number of users. Comparing the mean power levels of IUI (blue curves) with the ICI, both caused by the base stations' CFOs (red curves for $\xi=0$ ), we see that, independently of the number of users and for typical feedback delays between $2 \mathrm{~ms}$ and $10 \mathrm{~ms}$, the IUI lies between $40 \mathrm{~dB}$ and $50 \mathrm{~dB}$ above the power of ICI. This clearly indicates that the IUI is significantly stronger than the ICI caused by the base stations' CFOs. Considering now the second scenario with nonperfectly synchronized mobile users $(\xi=10)$, it can be observed that the part of the ICI caused by the users' CFO overwhelms the ICI caused by the base stations' CFOs. According to (38), the (dominant) ICI due to a mobile user's CFO does not depend

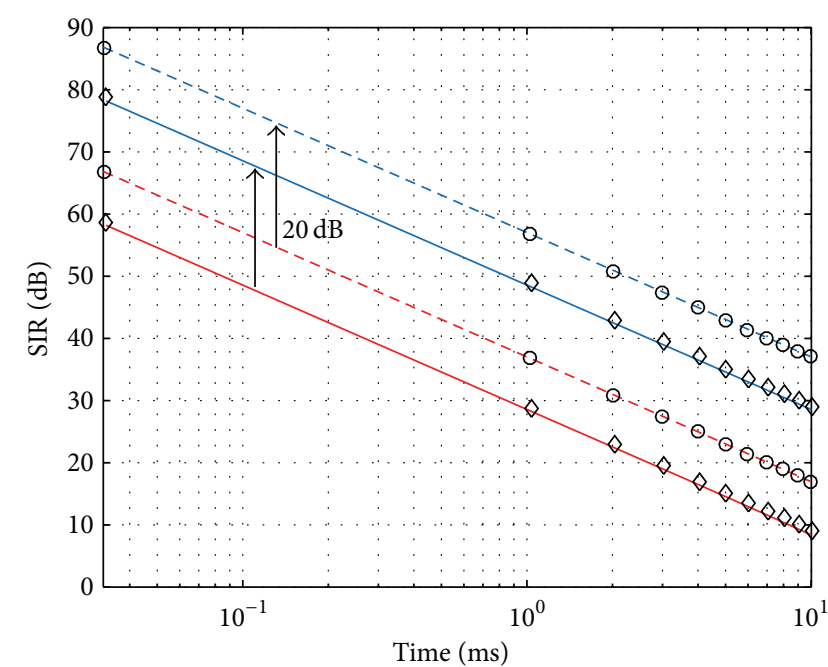

$$
\begin{array}{ll}
--\operatorname{SIR}_{\max }, \mathrm{Osc}=0.1 \mathrm{ppb} & --\mathrm{SIR}_{\max }, \mathrm{Osc}=1 \mathrm{ppb} \\
-\operatorname{SIR}_{\text {Rayleigh }}, \mathrm{Osc}=0.1 \mathrm{ppb} \quad-\mathrm{SIR}_{\text {Rayleigh }}, \mathrm{Osc}=1 \mathrm{ppb}
\end{array}
$$

FIGURE 3: Mean SIR over time for Rayleigh fading channel and SIR upper bound. Here, 7 base stations using oscillators of accuracy given by Osc serve jointly 6 users. Analytical results are shown by lines, simulations by markers.

on the number of served users, which is reflected in the fact that the black curves in Figure 2 evaluating the total ICI for $\xi=10$ almost overlap for the cases of 3 and 6 users. It is also interesting to observe that the IUI power level due to the cooperating base stations' CFOs is still around $20 \mathrm{~dB}$ to $30 \mathrm{~dB}$ (for 3 users) and $30 \mathrm{~dB}$ to $40 \mathrm{~dB}$ (for 6 users) higher than the total ICI power, considering typical feedback delays between $2 \mathrm{~ms}$ and $10 \mathrm{~ms}$. These results clearly show that in the a cooperative network, even with typically nonperfectly synchronized mobile users, the main interference source lies in the base stations' CFOs.

Figure 3 shows the SIR over the time, as defined in (41), for $N_{b}=7$ base stations serving jointly $N_{u}=6$ mobile users, which is also the highest possible number for the Rayleigh fading channel. The influence of the base stations' oscillator accuracy is evaluated, and the corresponding ICI is neglected as it is significantly smaller than the IUI. Disregarding the ICI also means that the synchronicity level of the mobile users is not relevant, as it does not contribute the IUI.

For the Rayleigh fading channel, the analytical expression (42) is compared with the ratio between the numerically evaluated mean power of (13) and (14) over $10^{3}$ independent channel and CFO realizations, which validates our analysis including the accuracy of the mathematical approximations. Similarly, for the SIR upper bound expression, the analytical expression (43) is compared with results from numerical evaluation. From Figure 3 it is seen that a degradation of the base station oscillators' accuracy by one order of magnitude increases the IUI and thus decreases the SIR by around $20 \mathrm{~dB}$. Furthermore, the SIR drops approximately quadratically with time, that is, $20 \mathrm{~dB}$ per time decade. In terms of accuracy requirements, it is found that, for reaching in average an SNR of $20 \mathrm{~dB} 10 \mathrm{~ms}$ after the precoder update, (free-running) oscillators of $1 \mathrm{ppb}$ are needed. 


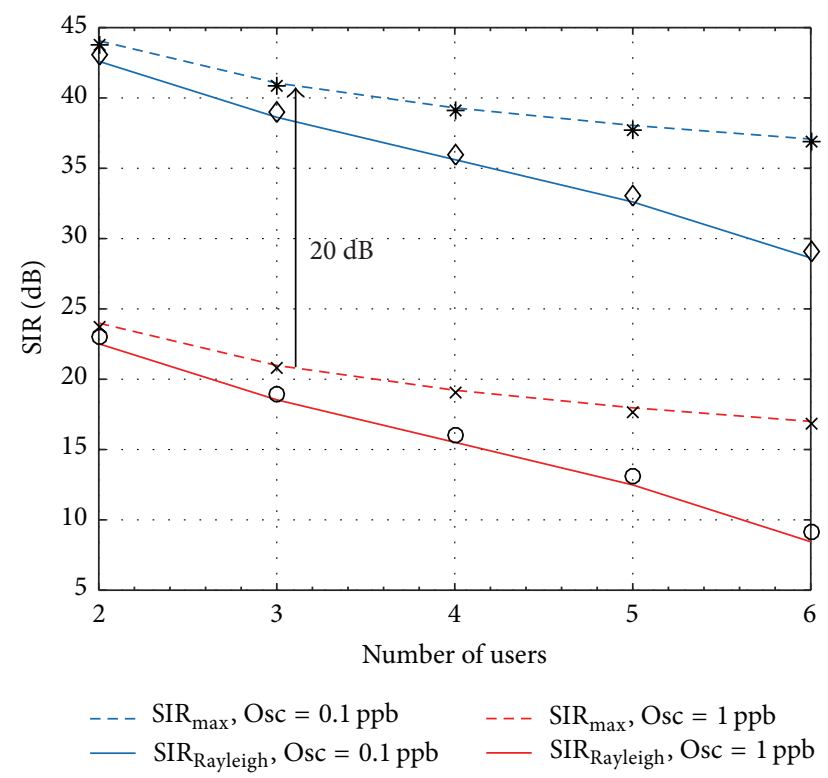

FIGURE 4: Mean users' SIR $10 \mathrm{~ms}$ after most recent precoder update, for the Rayleigh fading and upper bound. Here, 7 base stations serve jointly from 2 to 6 users. Analytical results are shown by lines, simulations by markers.

Figure 4 illustrates the mean SIR as function of the number of users, which are served by 7 base stations at the same time and frequency resource, for a time instant of $10 \mathrm{~ms}$ after the most recent precoder update. Such a time delay is relatively large for a practical system and thus these results should be interpreted more as a worst case rather than an average situation. Here, analytical results based on (41) for the Rayleigh fading channel and the SIR upper bound are verified by numerical simulations. It is observed that, given the same synchronization conditions, serving jointly more users results in a lower SIR compared to serving fewer users. It is also observed that, as the number of users grows, the distance between the SIR in Rayleigh fading and its upper bound becomes larger. Practically this means that the more the users that are jointly served, the higher the potential benefits if sets of jointly served users are appropriately formed, as already discussed in Section 4.5.

\section{Recommendations for CoMP System Design}

In this section, synchronization requirements for OFDMbased JT CoMP and ways to meet them are discussed. It can be seen from Figure 4 that if, for example, an average SIR of at least $25 \mathrm{~dB}$ will be guaranteed at all times between precoder updates and for the maximum allowed number of users, then the accuracy of the base stations' oscillators needs to be $0.1 \mathrm{ppb}$. Note that base stations typically contain already sufficiently precise oven-controlled crystal oscillators (OCXOs); however, their frequencies need to be locked to a common reference, which can be, for example, provided by the GPS. The satellites of the GPS system are equipped with Rubidium or Cesium oscillators, thus providing a highly reliable reference below $1 \mu \mathrm{s}$ in terms of absolute time error [37]. At each base station, equipped with a GPS receiver, the local oscillator will be then phase-locked to the incoming time signal from the GPS and will also follow its carrier frequency. Practical synchronization of distributed stations in a JT CoMP testbed is described in [27].

Other schemes, also for base stations with no GPS connection, suggest that base stations are connected via Ethernet to the backhaul network, over which, and by using a network synchronization protocol such as IEEE1588 [38], a common clock signal can be provided to them. This reference signal can be then used for time synchronization, as well as for recovering a carrier frequency reference at each site. However, the precision of such protocols could be probably not sufficient for JT CoMP. Therefore, additional over-theair synchronization procedures can be deployed, such as the protocol proposed in [28]. Distributed base stations incorporate a suitable frequency estimator, for example, the MLbased estimator described in [14], and additionally exchange pilots for enhancing the network synchronicity. The protocol can be also applied to networks with a large number of nodes and does not require expensive oscillators.

\section{Conclusions}

An exact signal model for multiuser multicellular systems using OFDM was derived, for transmissions impaired by individual carrier and sampling frequency offsets on every transmitter and receiver branch. The model was specialized to the downlink of systems with cooperative base stations, where precoding with the inverse of the channel matrix was considered. It was analyzed how carrier frequency misalignments among the cooperative base stations decrease the power of the self-user's signal and cause interuser and intercarrier interference. Closed-form exact expressions and accurate approximations were derived for the mean power of the above signals and it was shown that, for practical purposes, the interuser interference dominates on the intercarrier interference. Analytic expressions were also derived for the mean SIR, for Rayleigh fading channel conditions as well as an SIR upper bound for cooperative systems using zero-forcing precoding. The mean SIR decreases quadratically with time and is inversely proportional to the variance of the base stations' frequency offsets. It grows with the number of base stations and drops with the number of users jointly served by them on the same time and frequency resource. From a practical point of view, when a high SIR is targeted, synchronization requirements can be fulfilled by using at the base stations OCXOs locked either to a precise GPS reference or to an accurate clock signal provided through the backhaul network.

\section{Appendices}

\section{A. Analysis of $K_{\mathrm{U}}, K_{\mathrm{IUI}}$ and $K_{\mathrm{ICI}}$}

The constants $K_{\mathrm{U}}, K_{\mathrm{IUI}}$, and $K_{\mathrm{ICI}}$ are introduced in the main text in (26), (31), and (38), respectively. For identical channel statistics for all users and $K \triangleq \sum_{b=1}^{N_{b}} \mathbb{E}\left\{\left|H_{j b} w_{b u}\right|^{2}\right\}$, we have 
$K_{\mathrm{U}}=1-K, K_{\mathrm{IUI}}=\left(N_{u}-1\right) \cdot K$, and $K_{\mathrm{ICI}}=N_{u} \cdot K$. The following derivation gives an approximation for $K$ in Rayleigh fading. The correlation between $H_{j b}$ and $w_{b u}$ can be considered as weak, because taking into account how the pseudoinverse is calculated, there is only minor contribution of element $H_{j b}$ to $w_{b u}$. Thus,

$$
\begin{aligned}
K & =\sum_{b=1}^{N_{b}} \mathbb{E}\left\{\left|H_{j b} w_{b u}\right|^{2}\right\}=\sum_{b=1}^{N_{b}} \mathbb{E}\left\{\left|H_{j b}\right|^{2} \mathbb{E}\left\{\left|w_{b u}\right|^{2} \mid H_{j b}\right\}\right\} \\
& \approx \sum_{b=1}^{N_{b}} \mathbb{E}\left\{\left|H_{j b}\right|^{2}\right\} \mathbb{E}\left\{\left|w_{b u}\right|^{2}\right\}=\sigma_{h}^{2} \sum_{b=1}^{N_{b}} \mathbb{E}\left\{\left|w_{b u}\right|^{2}\right\},
\end{aligned}
$$

where $\mathbb{E}\{\cdot \mid A\}$ denotes the conditional expectation given event $A$. Using the analytical expressions provided by [39] for the sum term in the right-hand side of (A.1) for ZF precoding with $N_{b}>N_{u}$, we reach

$$
K \approx \frac{1}{N_{b}-N_{u}}, \quad K_{\min } \approx \frac{1}{N_{b}} .
$$

The first expression for $K$ in (A.2) refers to i.i.d. complex Gaussian random entries with zero mean and variance $\sigma_{h}^{2}$ and can be used to obtain expressions for $K_{\mathrm{U}}, K_{\mathrm{IUI}}$, and $K_{\mathrm{ICI}}$, while $K_{\min }$ is used for their bounds. Those can then be used in (26), (31), and (38) in the main text.

\section{B. Analysis of $B_{1}$ and $B_{2}$}

We analyze $\sum_{\nu=-N_{s} / 2, \nu \neq k}^{N_{s} / 2-1} \mathbb{E}\left\{\beta_{j b_{1}}(k, v) \beta_{j b_{2}}^{*}(k, v)\right\}$, which is given in (35) in the main text, while $\beta_{j b}(k, v)$ is given in (9). The base stations' and $j$ th mobile user's CFOs are considered as i.i.d. Gaussian-distributed with zero mean and variance $\sigma_{f}^{2}$ and $\sigma_{f, j}^{2}$, respectively.

For the first case $b_{1}=b_{2}=b$, we proceed as follows:

$$
\begin{aligned}
\sum_{\substack{\nu=-N_{s} / 2 \\
v \neq k}}^{N_{s} / 2-1}\left|\beta_{j b}(k, v)\right|^{2} & =\sum_{\nu=-N_{s} / 2}^{N_{s} / 2-1}\left|\beta_{j b}(k, v)\right|^{2}-\left|\beta_{j b}(k, k)\right|^{2} \\
& =1-\left|\beta_{j b}(k, k)\right|^{2} \\
& =1-\frac{1}{N_{s}^{2}} \cdot \frac{\sin ^{2}\left(\pi\left(f_{j}-f_{b}\right) T N_{s}\right)}{\sin ^{2}\left(\pi\left(f_{j}-f_{b}\right) T\right)} \\
& \approx \frac{\pi^{2}\left(f_{j}-f_{b}\right)^{2}}{3 \delta^{2}} \\
& =\frac{\pi^{2}\left[\left(f_{j}-f_{c}\right)-\left(f_{b}-f_{c}\right)\right]^{2}}{3 \delta^{2}} .
\end{aligned}
$$

From (B.1) to (B.2) we used the result (C.1) of Appendix C, which shows that the power of $N_{s}$ samples of a function $\alpha(k)=\left(1 / N_{s}\right)\left(\sin \left(N_{s} \Phi(k)\right) / \sin (\Phi(k))\right)$ with $\Phi(k)=\pi k / N_{s}$, taken at frequencies $k+\phi$, is equal to 1 for any offset $\phi \in \mathbb{R}$. Imposing (9) into (B.2) gives (B.3). For typical values, the Taylor series expansion $\left(1 / N_{s}^{2}\right)\left(\sin ^{2}\left(N_{s} x\right) / \sin ^{2}(x)\right) \approx 1-$ $(1 / 3) N_{s}^{2} x^{2}\left(N_{s}^{2}-1 \approx N_{s}^{2}\right.$ for $\left.N_{s} \gg 1\right)$ is accurate and was used in (B.3) to reach (B.4). Note that $\delta=\left(N_{s} T\right)^{-1}$ is the subcarrier spacing. Taking the expectation of (B.4), and considering the independence between $f_{j}$ and $f_{b}$, we reach

$$
\sum_{\substack{\nu=-N_{s} / 2 \\ \nu \neq k}}^{N_{s} / 2-1} \mathbb{E}\left\{\left|\beta_{j b}(k, v)\right|^{2}\right\} \approx \frac{\pi^{2}\left(\sigma_{f}^{2}+\sigma_{f, j}^{2}\right)}{3 \delta^{2}} \triangleq B_{1}
$$

For the second case $b_{1} \neq b_{2}$, we have

$$
\begin{aligned}
& \sum_{\substack{\nu=-N_{s} / 2 \\
\nu \neq k}}^{N_{s} / 2-1} \mathbb{E}\left\{\beta_{j b_{1}}(k, v) \beta_{j b_{2}}^{*}(k, v)\right\} \\
& =\frac{1}{N_{s}^{2}} \cdot\left|\mathscr{F}_{p}\left(t_{n}\right)\right|^{2} \\
& \quad \sum_{\substack{\nu=-N_{s} / 2 \\
\nu \neq k}}^{N_{s} / 2-1}\left|\mathbb{E}\left\{\frac{\sin \left[\pi(k-v)+\pi\left(f_{j}-f_{b}\right) T N_{s}\right]}{\sin \left[\left(\pi / N_{s}\right)(k-v)+\pi\left(f_{j}-f_{b}\right) T\right]}\right\}\right|^{2} .
\end{aligned}
$$

Expression (B.6) was numerically evaluated and it was found that for $f_{j}=f_{c}$ (perfectly synchronized mobiles) it is significantly smaller than (B.5). For $\sigma_{f, j} \geq \sigma_{f}$, (B.6) is given by

$$
\sum_{\substack{\nu=-N_{s} / 2 \\ \nu \neq k}}^{N_{s} / 2-1} \mathbb{E}\left\{\beta_{j b_{1}}(k, v) \beta_{j b_{2}}^{*}(k, v)\right\} \approx \frac{\pi^{2} \sigma_{f, j}^{2}}{3 \delta^{2}} \triangleq B_{2} .
$$

Expressions (B.5) and (B.7) provide accurate approximations for $B_{1}$ and $B_{2}$ for Gaussian-distributed CFOs. Those results allow for taking the step from (36) to (37) in the main text.

\section{Power of a Periodic Band-Limited Function Sampled with an Offset}

Consider the function $\alpha(x)=\left(1 / N_{s}\right) \sum_{n=-N_{s} / 2}^{N_{s} / 2-1} e^{j 2 \Phi(x) n}$ where $\Phi(x)=\pi x / N_{s}$. It can be seen that $\alpha(x)$ is a periodic bandlimited function with period $T=N_{s}$ and Nyquist frequency $1 / \Delta=1$, and that $\alpha(x)=\sin \left(N_{s} \Phi(x)\right) / N_{s} \sin (\Phi(x))$. We want to show that

$$
\sum_{k=0}^{N_{s}-1}|\alpha(k+\phi)|^{2}=\sum_{k=0}^{N_{s}-1}|\alpha(k)|^{2}=1
$$

for offset $\phi \in \mathbb{R}$. To prove this, we present the following lemma.

Lemma C.1. Let $\alpha(x)=\sum_{r=0}^{N-1} A_{r} \exp (j 2 \pi(r / T) x)$ be an arbitrary periodic band-limited function of period $T$ with 
Fourier coefficients $A_{r}$. Let one define $\gamma^{(\phi)}(x)$ by sampling $\alpha(x)$ with frequency $1 / \Delta$ and offset $\phi$ :

$$
\begin{aligned}
\gamma^{(\phi)}(x) & =\alpha(x+\phi)\left(\sum_{k=-\infty}^{\infty} \delta(x-k \Delta)\right) \\
& =\sum_{k=-\infty}^{\infty} \alpha_{k}^{(\phi)} \delta(x-k \Delta),
\end{aligned}
$$

where $\alpha_{k}^{(\phi)}=\alpha(k \Delta+\phi)$ and $\delta(\cdot)$ is the delta distribution. Then, if $1 / \Delta=N / T$ (which is the Nyquist frequency for $\alpha(x)$ ), the energy contained in one period of $\gamma^{(\phi)}$ is given by

$$
\sum_{k=0}^{N-1}\left|\alpha_{k}^{(\phi)}\right|^{2}=\frac{1}{\Delta} \int_{0}^{T}|\alpha(x)|^{2} d x
$$

and therefore is independent of $\phi$.

Proof. $\gamma^{(\phi)}$ is a periodic function for which we calculate Fourier coefficients $\Gamma_{r}^{(\phi)}$ in two ways.

First, if $\mathscr{F}\{f(x)\}(r)=1 / T \int_{0}^{T} f(x) \exp \{-\jmath 2 \pi r x / T\} d x$ denotes the Fourier transform for a periodic function $f(x)$, using (C.3), one obtains

$$
\begin{aligned}
\Gamma_{r}^{(\phi)} & =\left[\mathscr{F}\{\alpha(x+\phi)\}(s) * \mathscr{F}\left\{\sum_{k=-\infty}^{\infty} \delta(x-k \Delta)\right\}(s)\right](r) \\
& =\left[\exp \left\{j 2 \pi \frac{s}{T} \phi\right\} A_{s}\right] *\left[\frac{1}{\Delta} \sum_{u=-\infty}^{\infty} \delta_{s}^{u N}\right](r) \\
& =\frac{\exp \{j 2 \pi(r / T) \phi\}}{\Delta} A_{r},
\end{aligned}
$$

where $r \in \mathbb{Z},\left[a_{s} * b_{s}\right](r)=\sum_{s=-\infty}^{\infty} a_{s} b_{r-s}$ denotes the discrete linear convolution, $\delta_{s}^{x}$ is a sequence of numbers that has its sth entry equal to 1 if $s=x$ and zero otherwise, and $\check{r}$ is the smallest positive integer that satisfies $\check{r} \equiv r \bmod N$. If $r \in$ $\{0, \ldots, N-1\}$, then $\check{r}=r$.

Next, using (C.3) and the fact that $T=N \Delta$, the Fourier coefficient $\Gamma_{k}^{(\phi)}$ can also be found as

$$
\begin{aligned}
\Gamma_{r}^{(\phi)} & =\frac{1}{T} \int_{-\Delta / 2}^{T-\Delta / 2} \gamma^{(\phi)}(x) e^{-\jmath 2 \pi(r / T) x} d x \\
& =\frac{1}{T} \sum_{k=-\infty}^{\infty} \int_{-\Delta / 2}^{(N-1 / 2) \Delta} \alpha_{k}^{(\phi)} \delta(x-k \Delta) e^{-\jmath 2 \pi(r / T) x} d x \\
& =\frac{1}{T} \sum_{k=0}^{N-1} \alpha_{k}^{(\phi)} e^{-\jmath 2 \pi(r k / N)},
\end{aligned}
$$

where (C.8) holds because the integral of a periodic function over its full period is translation invariant. Equation (C.10) implies that $\Gamma_{r}^{(\phi)}$ is the discrete Fourier transform (DFT) of $\alpha_{k}^{(\phi)} / T$, and therefore the DFT Parseval identity says that

$$
\sum_{k=0}^{N-1}\left|\frac{\alpha_{k}^{(\phi)}}{T}\right|^{2}=\frac{1}{N} \sum_{k=0}^{N-1}\left|\Gamma_{r}^{(\phi)}\right|^{2} .
$$

Finally, using (C.7) with $\check{r}=r$ and (C.11), it can be proven that

$$
\begin{aligned}
\sum_{k=0}^{N-1}\left|\alpha_{k}^{(\phi)}\right|^{2} & =\frac{T^{2}}{N} \sum_{k=0}^{N-1}\left|\Gamma_{r}^{(\phi)}\right|^{2} \\
& =\frac{T^{2}}{N} \sum_{k=0}^{N-1}\left|\frac{\exp \{j 2 \pi(r / T) \phi\}}{\Delta} A_{r}\right|^{2} \\
& =N \sum_{k=0}^{N-1}\left|A_{r}\right|^{2} \\
& =\frac{N}{T} \int_{0}^{T}|\alpha(x)|^{2} d x,
\end{aligned}
$$

which is what we wanted to prove in (C.1). Above, we used the Parseval identity for Fourier series.

\section{Disclosure}

This work was done during Konstantinos Manolakis doctoral studies at the Technische Universität Berlin, Germany.

\section{Conflict of Interests}

The authors declare that there is no conflict of interests regarding the publication of this paper.

\section{Acknowledgments}

The authors would like to acknowledge the Deutsche Forschungsgemeinschaft (DFG) for support within project CoMP impairments (JU 2793/3-1) and CoMP mobil (JU 2793/4-1). This work was also supported by projects from CONICYT, Departamento de Relaciones Internacionales "Programa de Cooperación Científica Internacional" CONICYT/DFG-622 and FONDECYT 1110370. Fernando Rosas would also like to acknowledge support of the F+ fellowship from KU Leuven and the SBO project SINS, funded by the Agency for Innovation by Science and Technology IWT (Belgium). Finally, the authors would like to thank Dr. Malte Schellmann from the Huawei European Research Center for his useful comments and suggestions.

\section{References}

[1] M. Karakayali, G. J. Foschini, and R. A. Valenzuela, "Network coordination for spectrally efficient communications in cellular systems," IEEE Wireless Communications, vol. 13, no. 4, pp. 5661, 2006.

[2] G. J. Foschini, K. Karakayali, and R. A. Valenzuela, "Coordinating multiple antenna cellular networks to achieve enormous spectral efficiency," IEE Proceedings: Communications, vol. 153, no. 4, pp. 548-555, 2006.

[3] D. Gesbert, M. Kountouris, R. W. Heath, C.-B. Chae, and T. Sälzer, "Shifting the MIMO paradigm," IEEE Signal Processing Magazine, vol. 24, no. 5, pp. 36-46, 2007.

[4] Q. H. Spencer, A. L. Swindlehurst, and M. Haardt, "Zero-forcing methods for downlink spatial multiplexing in multiuser MIMO 
channels," IEEE Transactions on Signal Processing, vol. 52, no. 2, pp. 461-471, 2004.

[5] A. Goldsmith, S. A. Jafar, N. Jindal, and S. Vishwanath, "Capacity limits of MIMO channels," IEEE Journal on Selected Areas in Communications, vol. 21, no. 5, pp. 684-702, 2003.

[6] R. Irmer, H. Droste, P. Marsch et al., "Coordinated multipoint: concepts, performance, and field trial results," IEEE Communications Magazine, vol. 49, no. 2, pp. 102-111, 2011.

[7] F. Boccardi, B. Clerckx, A. Ghosh et al., "Multiple-antenna techniques in LTE-advanced," IEEE Communications Magazine, vol. 50, no. 3, pp. 114-121, 2012.

[8] D. Lee, H. Seo, B. Clerckx et al., "Coordinated multipoint transmission and reception in LTE-advanced: deployment scenarios and operational challenges," IEEE Communications Magazine, vol. 50, no. 2, pp. 148-155, 2012.

[9] V. Jungnickel, K. Manolakis, W. Zirwas et al., "The role of small cells, coordinated multipoint, and massive MIMO in 5G," IEEE Communications Magazine, vol. 52, no. 5, pp. 44-51, 2014.

[10] X. Tao, X. Xu, and Q. Cui, "An overview of cooperative communications," IEEE Communications Magazine, vol. 50, no. 6, pp. 65-71, 2012.

[11] G. J. Foschini and M. J. Gans, "On limits of wireless communications in a fading environment when using multiple antennas," Wireless Personal Communications, vol. 6, no. 3, pp. 311-335, 1998.

[12] Q. H. Spencer, C. B. Peel, A. L. Swindlehurst, and M. Haardt, "An introduction to the multi-user MIMO downlink," IEEE Communications Magazine, vol. 42, no. 10, pp. 60-67, 2004.

[13] M. Speth, S. A. Fechtel, G. Fock, and H. Meyr, "Optimum receiver design for wireless broad-band systems using OFDM. Part I," IEEE Transactions on Communications, vol. 47, no. 11, pp. 1668-1677, 1999.

[14] C. Oberli, "ML-based tracking algorithms for MIMO-OFDM," IEEE Transactions on Wireless Communications, vol. 6, no. 7, pp. 2630-2639, 2007.

[15] L. Häring, S. Bieder, A. Czylwik, and T. Kaiser, "Estimation algorithms of multiple channels and carrier frequency offsets in application to multiuser OFDM systems," IEEE Transactions on Wireless Communications, vol. 9, no. 3, pp. 865-870, 2010.

[16] M. Schellmann and V. Jungnickel, "Multiple CFOs in OFDMSDMA uplink: interference analysis and compensation," EURASIP Journal on Wireless Communications and Networking, vol. 2009, Article ID 909075, 14 pages, 2009.

[17] K. Manolakis, C. Oberli, and V. Jungnickel, "Synchronization requirements for OFDM-based cellular networks with coordinated base stations: preliminary results," in Proceedings of the 15th International OFDM-Workshop, Hamburg, Germany, September 2010.

[18] P. Marsch and G. Fettweis, Eds., Coordinated Multi-Point in Mobile Communications: From Theory to Practice, Cambridge University Press, 2011.

[19] R. Mudumbai, D. R. Brown, U. Madhow, and H. V. Poor, "Distributed transmit beamforming: challenges and recent progress," IEEE Communications Magazine, vol. 47, no. 2, pp. 102-110, 2009.

[20] T. Koivisto and V. Koivunen, "Impact of time and frequency offsets on cooperative multi-user MIMO-OFDM systems," in Proceedings of the IEEE 20th International Symposium on Personal, Indoor and Mobile Radio Communications (PIMRC '09), September 2009.
[21] T. Koivisto and V. Koivunen, "Low complexity estimation of multiple frequency offsets using optimized training signals," in Proceedings of the IEEE 11th International Symposium on Spread Spectrum Techniques and Applications (ISITA '10), pp. 81-86, Taichung, Taiwan, October 2010.

[22] B. W. Zarikoff and J. K. Cavers, "Multiple frequency offset estimation for the downlink of coordinated MIMO systems," IEEE Journal on Selected Areas in Communications, vol. 26, no. 6, pp. 901-912, 2008.

[23] Y. R. Tsai, H. Y. Huang, Y. C. Chen, and K. J. Yang, "Simultaneous multiple carrier frequency offsets estimation for coordinated multipoint transmission in OFDM systems," IEEE Transactions on Wireless Communications, vol. 12, no. 9, pp. 4558-4568, 2013.

[24] B. W. Zarikoff and J. K. Cavers, "Coordinated multi-cell systems: carrier frequency offset estimation and correction," IEEE Journal on Selected Areas in Communications, vol. 28, no. 9, pp. 14901501, 2010.

[25] V. Kotzsch and G. Fettweis, "Interference analysis in time and frequency asynchronous network MIMO OFDM systems," in Proceedings of the IEEE Wireless Communications and Networking Conference (WCNC '10), pp. 1-6, April 2010.

[26] D. R. Brown III and H. V. Poor, “Time-slotted round-trip carrier synchronization for distributed beamforming," IEEE Transactions on Signal Processing, vol. 56, no. 11, pp. 5630-5643, 2008.

[27] V. Jungnickel, T. Wirth, M. Schellmann, T. Haustein, and W. Zirwas, "Synchronization of cooperative base stations," in Proceedings of the IEEE International Symposium on Wireless Communication Systems (ISWCS '08), pp. 329-334, October 2008.

[28] R. Rogalin, O. Y. Bursalioglu, H. Papadopoulos et al., "Scalable synchronization and reciprocity calibration for distributed multiuser MIMO," IEEE Transactions on Wireless Communications, vol. 13, no. 4, pp. 1815-1831, 2014.

[29] O. Simeone, U. Spagnolini, Y. Bar-Ness, and S. H. Strogatz, "Distributed synchronization in wireless networks," IEEE Signal Processing Magazine, vol. 25, no. 5, pp. 81-97, 2008.

[30] C. Oberli, "Erratum to 'ML-based tracking algorithms for MIMO-OFDM', IEEE Transactions on Wireless Communications, vol. 6, no. 7, pp. 2630-2639, 2007," September 2007.

[31] 3rd Generation Partnership Project, TS 36.300, V11.7.0, Evolved Universal Terrestrial Radio Access (E-UTRA) and Evolved Universal Terrestrial Radio Access Network (E-UTRAN); Overall Description, 3GPP Standards, 2013.

[32] A. Papoulis, Probability, Random Variables and Stochastic Processes, McGraw-Hill, New York, NY, USA, 2nd edition, 1991.

[33] F. Rosas, L. Herrera, C. Oberli, K. Manolakis, and V. Jungnickel, "Downlink performance limitations of cellular systems with coordinated base stations and mismatched precoder," IET Communications, vol. 8, no. 1, pp. 77-82, 2014.

[34] M. Lossow, S. Jaeckel, V. Jungnickel, and V. Braun, "Efficient MAC protocol for JT CoMP in small cells," in Proceedings of the IEEE 2nd International Workshop on Small Cell Wireless Networks (SmallNets '13), June 2013.

[35] K. Seong, M. Mohseni, and J. M. Cioffi, "Optimal resource allocation for OFDMA downlink systems," in Proceedings of the IEEE International Symposium on Information Theory, pp. 13941398, July 2006.

[36] S. Shi, M. Schubert, and H. Boche, "Rate optimization for multiuser MIMO systems with linear processing," IEEE Transactions on Signal Processing, vol. 56, no. 8, pp. 4020-4030, 2008. 
[37] M. A. Lombardi, L. M. Nelson, A. N. Novick, and V. S. Zhang, "Time and frequency measurements using the global positioning system," Cal Lab: International Journal of Metrology, vol. 8, no. 3, pp. 26-33, 2001.

[38] IEEE, "IEEE standard for a precision clock synchronization protocol for networked measurement and control systems," Tech. Rep. 1588-2008, IEEE Standards Association, 2008.

[39] K. Manolakis, C. Oberli, and V. Jungnickel, "Random matrices and the impact of imperfect channel knowledge on cooperative base stations," in Proceedings of the IEEE 14th International Workshop on Signal Processing Advances in Wireless Communications (SPAWC '13), pp. 480-484, Darmstadt, Germany, June 2013. 

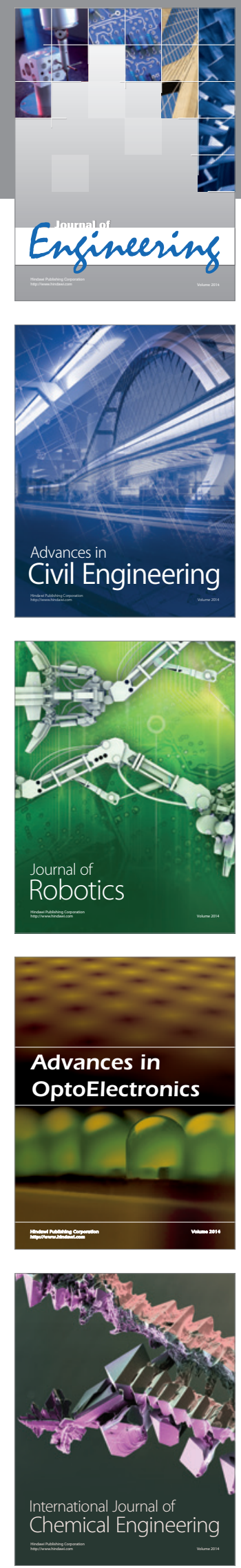

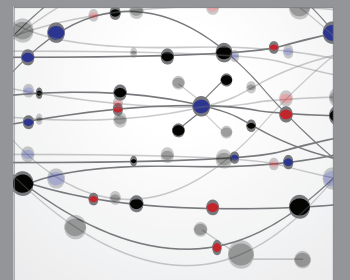

The Scientific World Journal
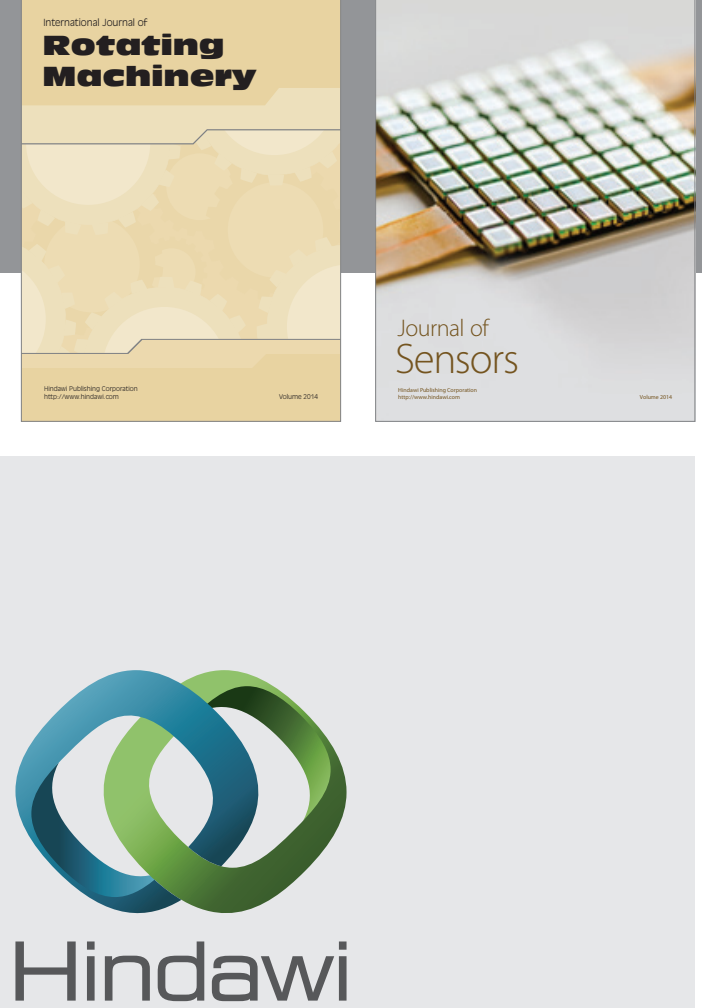

Submit your manuscripts at http://www.hindawi.com
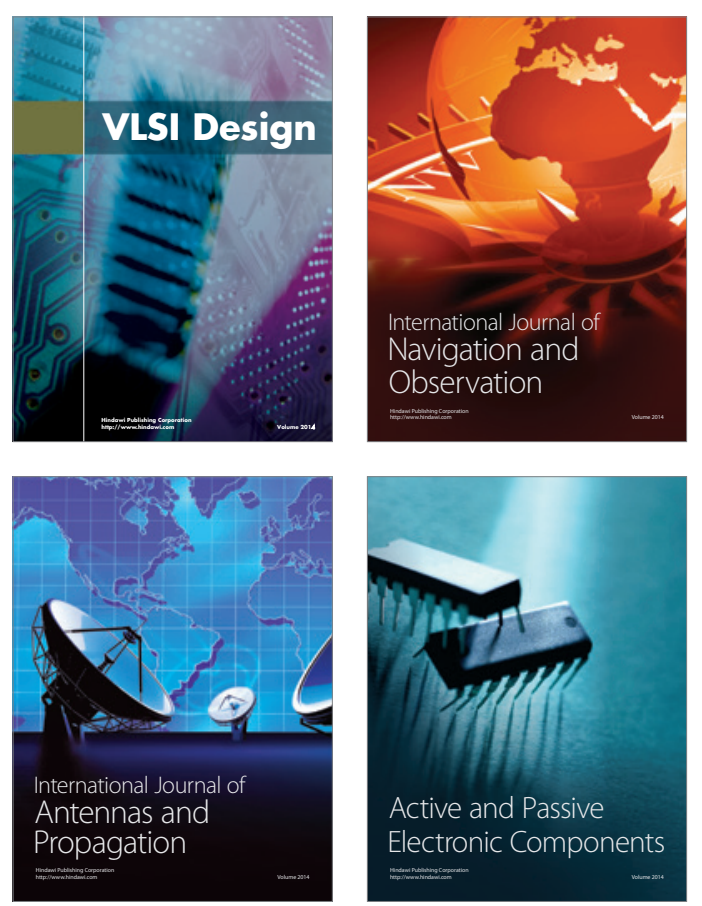
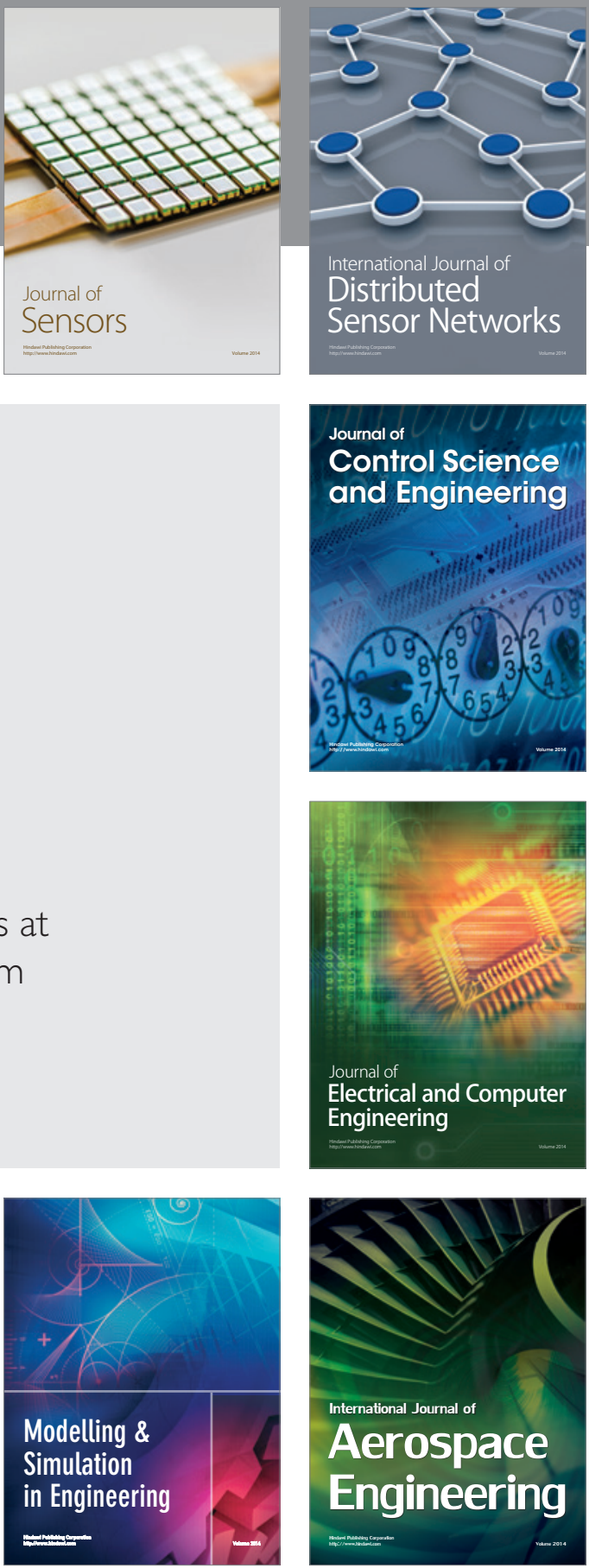

Journal of

Control Science

and Engineering
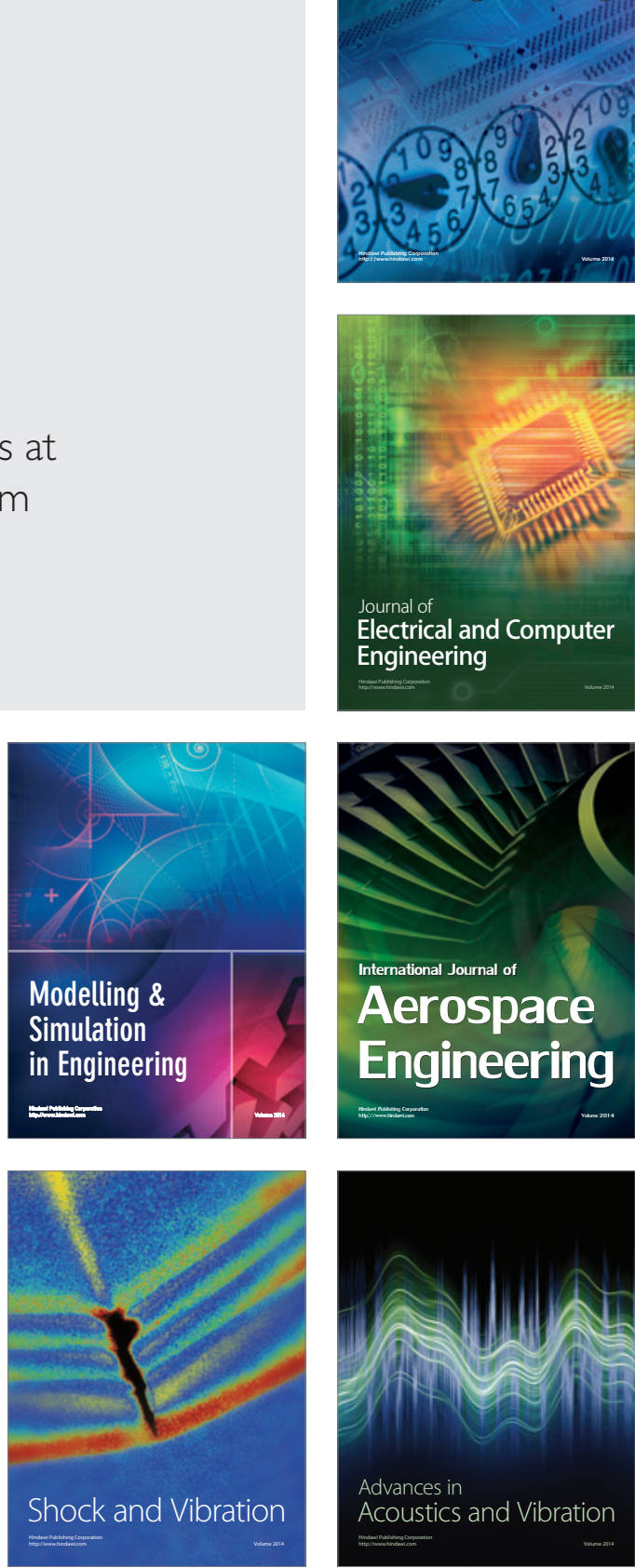